\title{
Application of Nanofiber Fabricated by Cotton Candy Method to Electric Double-Layer Capacitor
}

\section{Akihiro Tada', Tomohiko Adachi², Yoshiki Tanaka², Yoshifumi Aoi ${ }^{3}$, Atsushi Yokoyama4, Hiroyuki Hamada ${ }^{4}$}

${ }^{1}$ Ohgi Technological Creation Co., Ltd, Shiga, Japan

${ }^{2}$ Industrial Resarch Center of Shiga Prefecture, Shiga, Japan

${ }^{3}$ Ryukoku University, Kyoto, Japan

${ }^{4}$ Kyoto Institute of Technology, Kyoto, Japan

Email: tada.akihiro@ohki-techno.com

How to cite this paper: Tada, A., Adachi, T., Tanaka, Y., Aoi, Y., Yokoyama, A. and Hamada, H. (2018) Application of Nanofiber Fabricated by Cotton Candy Method to Electric Double-Layer Capacitor. Open Journal of Composite Materials, 8, 138-144. https://doi.org/10.4236/ojcm.2018.83011

Received: June 15, 2018

Accepted: July 27, 2018

Published: July 30, 2018

Copyright $\odot 2018$ by authors and Scientific Research Publishing Inc. This work is licensed under the Creative Commons Attribution International License (CC BY 4.0).

http://creativecommons.org/licenses/by/4.0/

\begin{abstract}
In recent years, application of carbon-based nano material to electrode material has been paid attention, however, due to its higher cost, it would be difficult to put it into practical use. Then, we have proposed to make nano carbon fiber with lower production cost. The purpose of our research was, to apply our nano carbon fiber to electrical double-layer capacitor electrode. We used cotton candy method to make nano fiber, and applied microwave heating for carbonization. By applying nano carbon fiber to electrical double-layer capacitor electrode, we got results that thicker electrode containing nano carbon fiber leads to lower resistance value, compared with electrode without containing nano carbon fiber. From this result, it was indicated that by containing nano carbon fiber, the electric bypass was formed in the electrode.
\end{abstract}

\section{Keywords}

Cotton Candy Method, Carbon Nanofiber, Electric Double Layer Capacitor

\section{Introduction}

The carbon-based nano materials, like fullerene, carbon nanotube, and graphene, have been paid attention, and they are applied to like electrode materials [1] [2].

The researches of applying carbon-based nano materials to electrode have been observed actively.

However, the practical application must be difficult as the cost of carbon-based nano materials for electrode is extremely high. The reason will be the 
higher production cost of carbon-based nano materials due to using bottom-up production method like CVD method.

Then, we have proposed to produce nano carbon fiber with lower production cost.

This is the production method of carbon-based nano materials by forming nano fiber and firing. We have developed cotton candy method, the new production method of nano fiber. And we have decided to use microwave heating method for firing. Polyacrylonitrile are generally produced into micro and nano fibers by dry or wet spinning and electrospinning etc. [3] [4] [5] [6]. The examples of polyacrylonitrile fibers are made by solution or dry-jet wet spinning. Because polyacrylonitrile is the high polarity of nitrile groups makes the polymer infusible [5] [6]. Polyacrylonitrile becomes carbon fiber by infusibilization process and carbonization process. Using the cotton candy method, the nano fiber is gushed from unique shaped nozzle without using electric field or heating. This method realizes mass production of nano fiber, thus the production cost will be reduced [7] [8]. In addition, nanofibers made by the cotton candy method are very long fibers.

Microwave heating method can rise the target temperature from inside, so the required time of rising temperature will be reduced.

At this research, we have aimed to apply nano fiber produced by this method to electrical double-layer capacitor electrode.

\section{Experiment}

\subsection{The Production of Nano Fiber and Carbonization of Nano Fiber}

We made nanofibers using plunger type cotton candy method. Nano fiber was produced by cotton candy method using mixed solvent of $\mathrm{N}$-methyl pyrrolidone and polyacrylonitrile (Mw: 150,000) with concentration of $10 \mathrm{wt} . \%$, discharging pressure of $0.2 \mathrm{M} \mathrm{Pa}$ and capturing distance of $40 \mathrm{~cm}$. Nanofiber preparation was performed at room temperature. Then remove the solvent from captured nano fiber by air drying. Regarding evaluation and equipment, at infusibilization, we used MMF-2F for electric furnace and WMW-144 for microwave heating furnace. The microwave frequency was $2450 \mathrm{MHz}$, the output was variable and heated. The firing of nano fiber has been done by microwave and electric furnace, processing with two steps. The first step was infusibilizing treatment, heating in electric furnace at 270 degrees Celsius for one hour in air atmosphere. The temperature increasing speed was 5 degrees Celsius per minute. The second step is carbonization, firing by microwave at 1400 degrees Celsius for 10 minutes in nitrogen atmosphere. The temperature increasing speed was 50 degrees Celsius per minute.

\subsection{The Production Method of Electrical Double-Layer Capacitor Electrode}

We have produced coin cell type capacitor, using activated carbon (Ohgi-made, 
made from PET waste, $2600 \mathrm{~m}^{2} / \mathrm{g}$ of specific surface area) as slurry of electrode, plane status of aluminum foil for collector and CMC for dispersant. The nanofibers were dispersed in ethanol and mixed in the slurry. We have produced slurry using carbon black for conductive auxiliary agent and SBR for binder, blending nano carbon fiber to content rate of $0,2,5 \mathrm{wt} . / \%$, applied collector on to electrode controlling the thickness by applicator setting.After applying the slurry, vacuum drying was performed at 100 degrees Celsius. After that, filling was carried out by pressing to prepare an electrode sheet. The electrode sheet was placed in a coin cell together with an electrolytic solution and a separator in grove box. We have formed coin cell type capacitor using $1 \mathrm{M} \mathrm{TEMABF}_{4} / \mathrm{PC}$ as electrolyte.

\subsection{Characterization}

We used MODEL:ESCA-1600MR for X-ray photoelectron spectroscopy (XPS) by using $\mathrm{MgK} a, 1256.3 \mathrm{eV}$ X-ray. RMP-510RS was used for Raman scattering spectroscopy (Raman) measurement. Measurement status was laser wavelength $523 \mathrm{~nm}$ and $5 \mathrm{~s}$ exposure time, 9 times accumulation count. JSM-6510LV and JSM-6010LA for scanning electron microscope (SEM) for evaluation.

And we used Solartron 1260 for impedance measurement and 585 Battery Test System (Scribner Associates) for charge/discharge measurement.Impedance measurement was carried out after 5 cycles of CC charging/discharging between 0 to $2.5 \mathrm{~V}$ at $5 \mathrm{~mA}$. Internal resistance measurement, $\mathrm{CC}$ charging/discharging was carried out at 0 to $2.5 \mathrm{~V}$ at $5 \mathrm{~mA} / \mathrm{cm}^{2}$.

\section{Result and Discussion}

\subsection{Characterization of Nanofiber and Nano Carbon Fiber}

Figure 1 is the SEM image of nano fiber produced by cotton candy method.

From above SEM image, the nano fiber produced by cotton candy method keeps fiber-form.

We used nano fiber with average diameter of approx. $460 \mathrm{~nm}$.

Figure 2 is the SEM image of nano fiber carbonized by microwave.

From Figure 2, the nano fiber carbonized by microwave firing keeps fiber-foam, and the average diameter of fiber was approx. $440 \mathrm{~nm}$.

We have confirmed the carbonization status of nano fiber by reviewing integrated status of carbon and nitrogen by XPS.

Figure 3 is the C1s and N1s spectra developed by XPS.

The C1s spectra of XPS show the bonding state of carbon. And, N1s spectra show the bonding state of nitrogen.From Figure 3, we could conclude that the nano carbon produced by this research composed by mainly carbon, and nitrogen containing in polyacrylonitrile is considered to be desorbed during the firing as we can confirm the N1s peaks rarely. This indicates that the carbonization has been performed normally.

We have confirmed the carbon status of the nano carbon fiber by measuring by Raman scattering spectroscopy. 


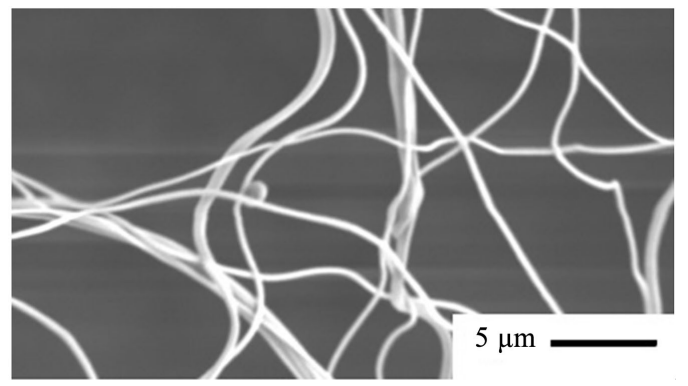

Figure 1. SEM image nanofiber by cotton candy method.

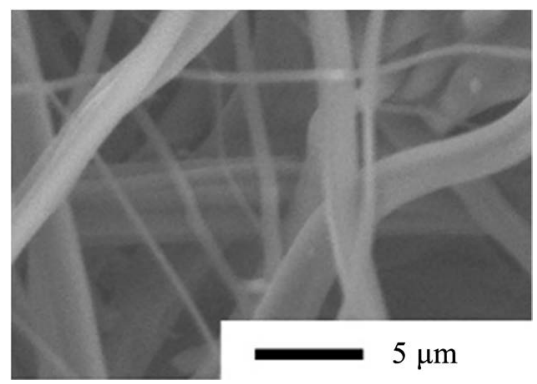

Figure 2. SEM image nano carbon fiber by micro wave heating.

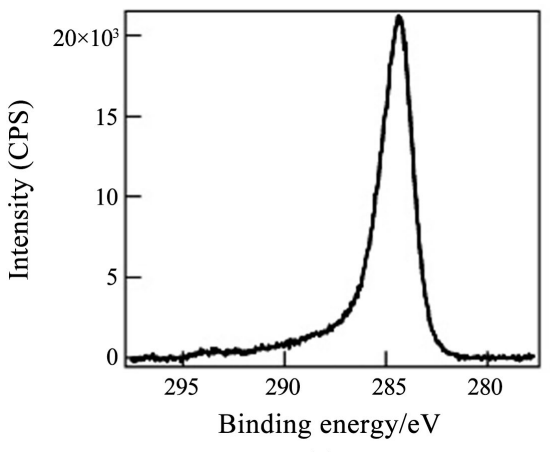

(a)

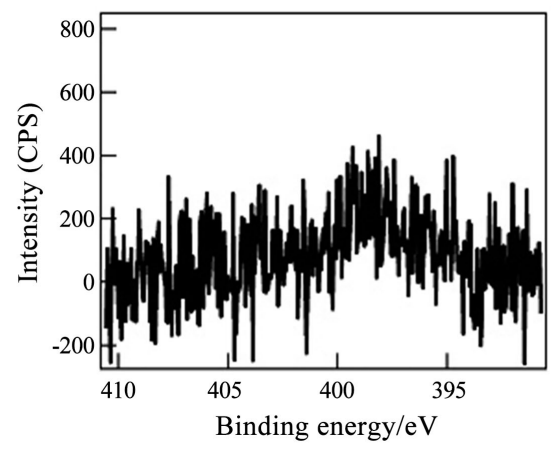

(b)

Figure 3. XPS spectra of nano carbon fiber (a) C1s spectra; (b) N1s spectra.

Figure 4 is the results of Raman scattering spectroscopy.

From measuring results by Raman scattering spectroscopy, we can observe two peaks, G (graphitic)-peak showing near $1570 \mathrm{~cm}^{-1}$ which is belonging to graphite structure peculiar to carbon material, and D (disorder)-peak showing near $1360 \mathrm{~cm}^{-1}$ which is belonging to disordered carbon structure.

We have calculated $I(D) / I(G)$ from peak ratio of D-peak and G-peak of Raman spectra as the variation of its figure is important parameter of finding out of carbon structure.

The $I(D) / I(G)$ value of the nano fiber was approx. 2.3, this figure is almost same as that of nano carbon fiber produced by electric furnace.

This result indicates that firing by microwave has no problems to make nano carbon fiber.

Then we have applied this nano carbon fiber to electrical double-layer capacitor. 


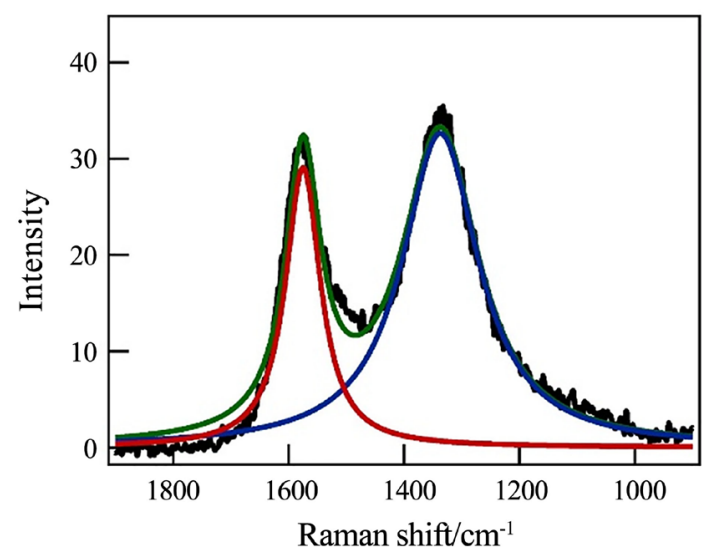

Figure 4. Raman spectra of nano carbon fiber.

\subsection{Application of Nano Carbon Fiber Produced By Cotton Candy Method to Electrical Double-Layer Capacitor}

Figure 5 shows the nano carbon fiber produced by cotton candy method and microwave heating at cross-section of electrical double-layer capacitor electrode.

From Figure 5, we can observe that the nano carbon has been dispersed in electrode, and this indicates that the conducting circuit has been formed inside of electrode.

Figure 6 shows the relationship between electrode thickness and internal resistance.

From Figure 6, we can observe that the electrode without containing nano carbon fiber has lower internal resistance value in case the thickness is less than 75 micrometers.

However, the electrode containing nano carbon fiber has lower internal resistance in case the thickness is over 75 micron meters. The NCF addition effect was considered to form a conductive path. In the case of normal carbon black it is a connection of conductive paths like stepping stones. When the electrode thickness is thin, there are two reasons why the internal resistance is high when NCF is added. First, since the electrode thickness is thin, NCF are lined up in the thickness direction. Therefore, since the conductive path in the thickness direction did not work effectively, the internal resistance increased. Second, contact resistance of NCF-activated carbon is larger than carbon black activated carbon. Contact than the effect of the thickness direction of the conductive path resistance is large, therefore, the internal resistance increased. In the electrode is thick case, effect of the conductive path becomes larger than the contact resistance. The effect was exerted as the electrode thickness became larger.

By containing nano carbon fiber, the electric resistivity among activated carbon will be considered to reduce, so, we have confirmed the resistance value by measuring impedance of electrode.

Figure 7 is the result of impedance measurement.

From Figure 7, we can observe that the hemicycle of cole-cole plot is getting smaller as increasing of variance. 


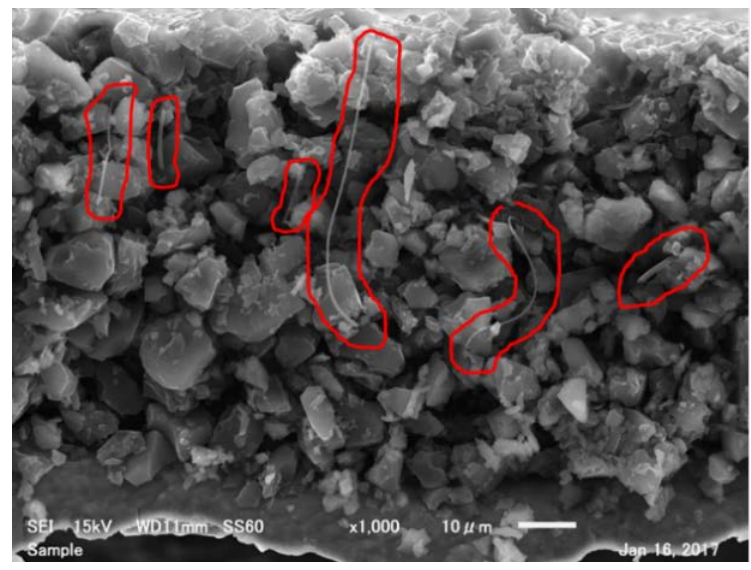

Figure 5. SEM image EDLC electrode.

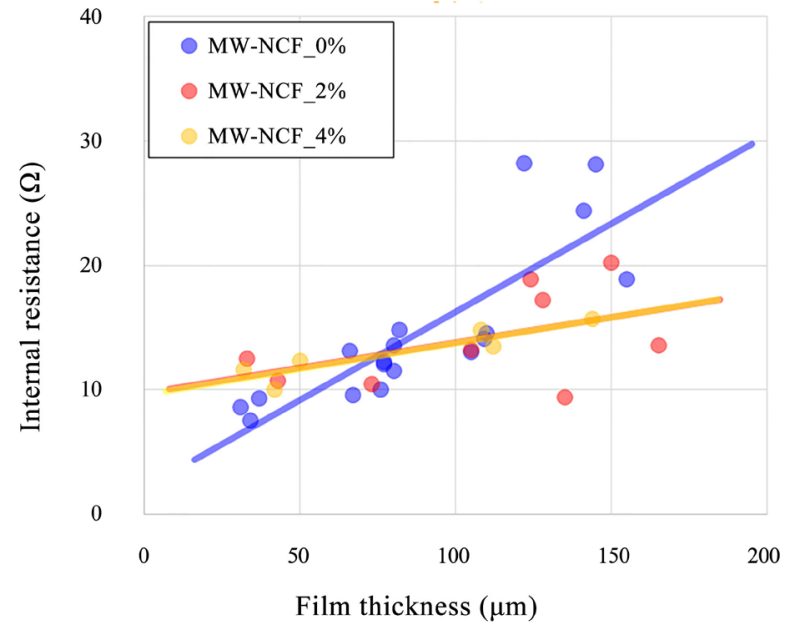

Figure 6. Relationship of resistance and thickness.

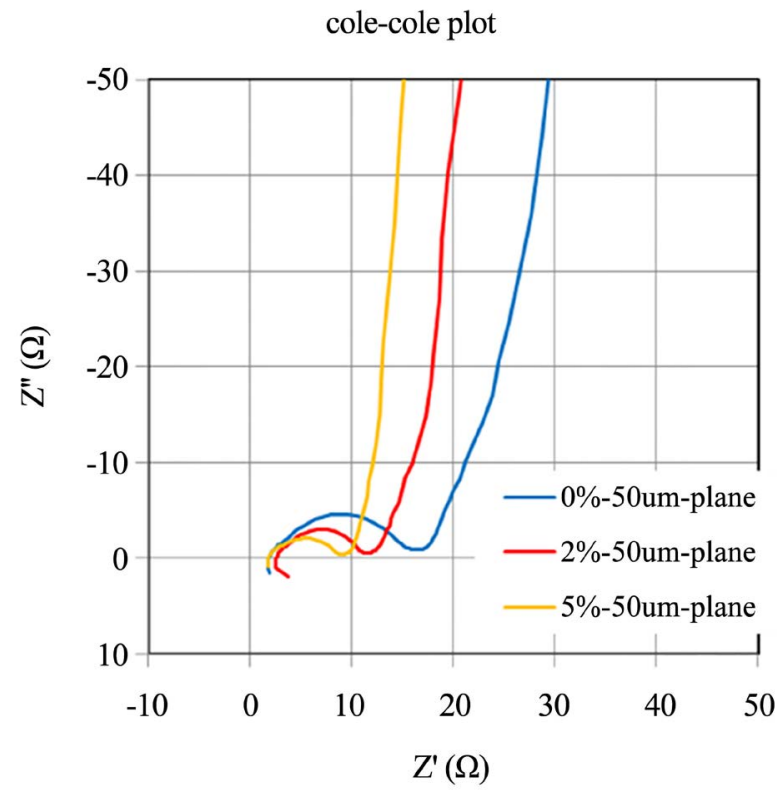

Figure 7. Cole-cole plot. 
The hemicycle is considered as showing contact resistance, then, we considered that the nano carbon fiber has decreasing effect of reducing electrical resistivity of electrode. This assumed that the nanocarbon fiber plays the role of bypass connecting activated carbon. From above, it is suggested that the nano carbon fiber produced by cotton candy method and microwave heating has possibility to apply to electrical double-layer capacitor.

\section{Conclusions}

We have produced nano carbon fiber using cotton candy method and microwave heating.

The $\mathrm{I}(\mathrm{D}) / \mathrm{I}(\mathrm{G})$ value of that nano carbon fiber was approx. 2.3, and have almost same characteristic as nano carbon fiber produced by electric furnace.

We have applied our nano carbon fiber to electrical double-layer capacitor.

Comparing with electrode without containing nano carbon fiber, we have confirmed that the electrical resistivity will decrease at thicker electrode.

From this, we have also confirmed that electrical bypass will be formed inside electrode by applying nano carbon fiber.

From above, it is suggested that the nano carbon fiber produced by cotton candy method and microwave heating has possibility to apply to electrical double-layer capacitor.

\section{References}

[1] Endo, M. and Iijima, S. (2007) Handbook of Nano Carbon. NTSinc, Tokyo.

[2] Suda, Y., Tanoue, H. and Takikawa, H. (2012) New Development of Carbon Nanomaterials. Journal of Plasma and Fusion Research, 88, 629.

[3] Jia, Z., Lu, C., Zhou, P. and Wang, L. (2016) Lignin/Polyacrylonitrile Composite Hollow Fibers Prepared by Wet-Spinning Method. ACS Sustainable Chemistry \& Engineering, 4, 2838-2842. https://doi.org/10.1021/acssuschemeng.6b00351

[4] Dong, X.-G., Wang, C.-G., Bay, Y.-J. and Cao, W.-W. (2007) Effect of DMSO/ $\mathrm{H}_{2} \mathrm{O}$ Coagulation Bath on the Structure and Property of Polyacrylonitrile Fibers during Wet-Spinning. Journal of Applied Polymer Science, 105, 1221-1227. https://doi.org/10.1002/app.25665

[5] Bajaj, P., Sreekumar, T.V. and Sen, K. (2002) Structure Development during Dry-Jet-Wet Spinning of acrylonitrile/vinyl Acids and acrylonitrile/methyl acrylate Copolymers. Journal of Applied Polymer Science, 86, 773-787. https://doi.org/10.1002/app.10973

[6] Tan, L.L., Chen, H., Pan, D. and Pan, N. (2008) Investigating the Spinnability in the Dry-Jet Wet Spinning of PAN Precursor Fiber. Journal of Applied Polymer Science, 110, 1997-2000. https://doi.org/10.1002/app.28029

[7] Wongpajan, R., Thumsorn, S., Inoya, H., Okoshi, M. and Hamada, H. (2016) Modern Fabrication of Poly(Lactic Acid) Nanofibers by Cotton Candy Method. SPE ANTEC Indianapolis 2016 Proceedings, 72, 260-264.

[8] Takematsu, R., Okoshi, M., Inoya, H., Hamada, H., Tada, A. and Aoi, Y. (2016) Study on Nano Polyacrylonitrile Fiber by Cotton Candy Method. SPE ANTEC Indianapolis 2016 Proceedings, 72, 542-546. 